\title{
Efficient synthesis of 2-alkoxy-1,3-butadienes by methylenation of alkenyl Fischer carbene complexes
}

\author{
José Barluenga,* Rubén Vicente, Luis A. López, and Miguel Tomás \\ Instituto Universitario de Química Organometálica "Enrique Moles”, Unidad Asociada al \\ CSIC, Universidad de Oviedo, Julián Clavería 8, 33071 Oviedo, Spain \\ E-mail: barluenga@uniovi.es
}

\begin{abstract}
The thermal reaction of chromium alkenyl carbene complexes $\mathbf{1}$ and 1,1dimethylpropadiene 2 results in the efficient formation of achiral and chiral 2-alkoxy-1,3butadienes 4. This process represents a new protocol for the methylenation of $\alpha, \beta$-unsaturated Fischer carbene complexes.
\end{abstract}

Keywords: Fischer carbene complexes, methylenation, 2-alkoxy-1,3-butadiene synthesis

\section{Introduction}

Heterosubstituted 1,3-butadienes have found numerous applications in the synthesis of functionalized cyclohexene derivatives by cycloaddition reactions. ${ }^{1}$ While a number of methodologies for the synthesis of achiral and, to a much lesser extent, chiral 2-amino-1,3butadienes have been reported, ${ }^{2}$ the corresponding 2-alkoxy-1,3-butadienes have been scarcely investigated, probably due to the difficulties associated with their preparation. While classical routes appear rather toilsome to access common 2-alkoxy-1,3-butadienes, sometimes they become unsuitable for derivatives with higher structural complexity, particularly chiral 2-alkoxy1,3-dienes. ${ }^{3}$ Thus, we found that these systems could be synthesized in moderate yields in two ways (Scheme 1): i) reaction of propargylphosphonium salts with chiral alcohols followed by the Wittig olefination (Route A), and ii) methylenation of carboxylic acid derivatives with dimethyltitanocene (Petasis reagent) (Route B). ${ }^{4}$

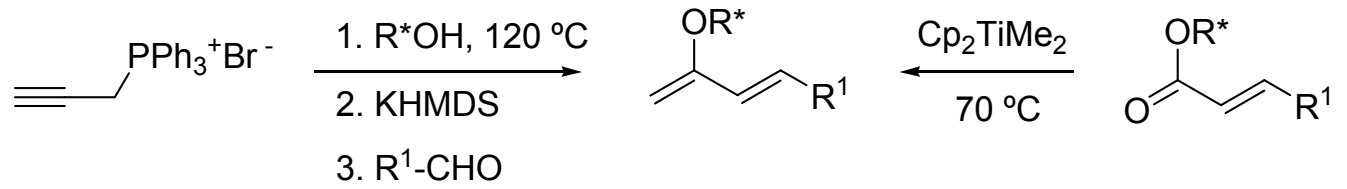

$($ via A)

(via B)

Scheme 1. Reported approaches for the synthesis of chiral 2-alkoxydienes. 
Although, the methylenation reaction represents a straightforward entry into such dienes, the problems associated with the titanium-based reagent prompted us to design a more efficient methylenation reaction. Since carbene-type substrates are well known to readily undergo methylenation, we focused our attention onto transition-metal alkoxycarbene complexes (Fischer carbene complexes) as more appropriate precursor systems of alkoxydienes. Their attractiveness seems obvious in terms of stability, availability in multigram scale, and reactivity. ${ }^{5}$ In this context, the rapid methylenation of simple carbene complexes has been reported with diazomethane $^{6}$ and chloromethyllithium ${ }^{7}$ (Scheme 2, eq. 1). On the contrary, severe problems arise when the methylenation reaction is extended to alkenyl carbene complexes, being the conjugate addition the major drawback particularly for carbene complexes containing bulky alkoxy groups. This is consistent with the fact that all the methylenation attempts on chiral alkenylcarbene complexes with diazomethane and chloromethyllithium resulted exclusively in the dipolar [3+2]- and [2+1]-cycloaddition reactions, respectively (Scheme 2, eq. 2). ${ }^{8,9}$<smiles>[R]C(OC)=C([O-])[C-](NC)OC</smiles>
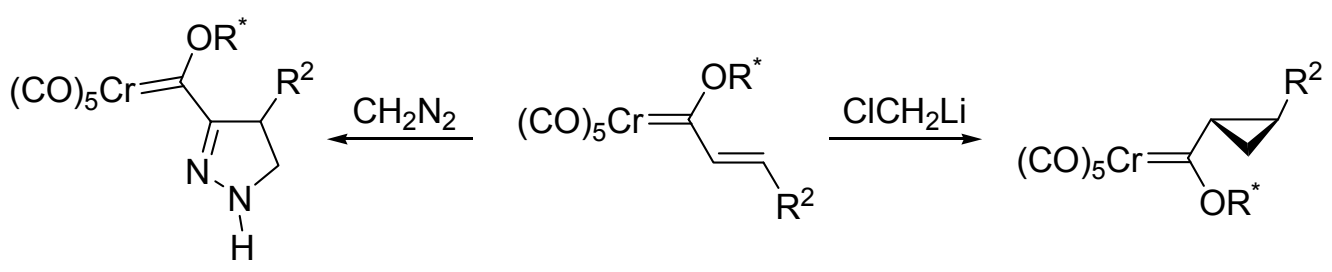

Scheme 2. The reaction of Fischer carbene complexes with methylenating reagents.

Herein we report that commercially available dimethylallene thermally reacts with achiral and chiral alkoxycarbene complexes of chromium to produce high yields of 2-alkoxy-1,3-dienes. This is the first metathesis reaction of transition-metal carbenes with allenes hitherto reported. ${ }^{10}$ This result complements our own results on the rhodium-catalyzed and nickel-mediated reactions of allenes and Fischer carbene complexes which provide five- and seven-membered carbocycles. $^{11}$

\section{Results and Discussion}

We started by treating the chromium carbene complex $1 \mathbf{a}\left(\mathrm{M}=\mathrm{Cr}, \mathrm{R}^{1}=p-\mathrm{CH}_{3} \mathrm{OC}_{6} \mathrm{H}_{4} ; \mathrm{R}^{2}=\mathrm{Me}\right)$ with dimethylallene 2a $\left(\mathrm{R}^{3}=\mathrm{R}^{4}=\mathrm{Me}, 3\right.$ equiv) in THF at $60^{\circ} \mathrm{C}$ (Table 1, entry 1$)$. Gratifyingly, 4-(4-methoxyphenyl)-3-buten-2-one 3a was formed in 63\% yield after stirring for $2 \mathrm{~h}$ and silica gel chromatographic purification. Therefore, the methylenation reaction is followed, under the purification conditions, by the enol ether hydrolysis to furnish the corresponding enone. Then 
some reaction variables, e. g. solvent, allene, and metal carbene, were checked. It was found that, although a number of solvents gave satisfactory results (entries 1-4), the best yield for compound 3a was achieved with 1,2-dichloroethane (DCE) (entry 4). Similar yields were reached for phenyl, 4-chlorophenyl and ferrocenyl substituted carbene complexes (entries 5-7). Remarkably, the methylenation/hydrolysis sequence of a metal carbene having a bulky alkoxy group occurred with the highest yield (entry 8). The use of 1,1-diphenylallene (entries 9 and 10) and phenylallene (entry 11) was unsuccessful as lower yield and a complex mixture, respectively, were obtained. Lastly, the chromium carbene complexes led to significant higher yields than their tungsten analogs (entry 5 vs entry 12).

Table 1. Thermal reaction of alkenyl carbene complexes $\mathbf{1}$ and allenes $\mathbf{2}$

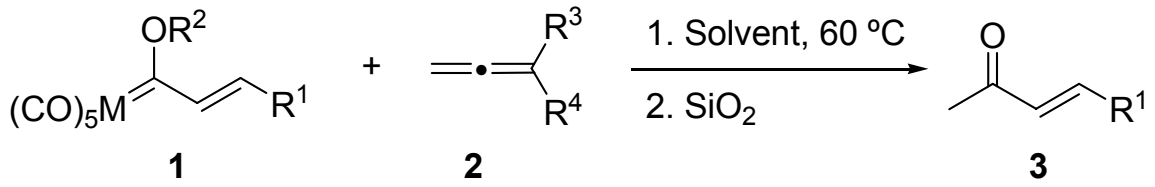

\begin{tabular}{llllllll}
\hline Entry & $\mathrm{M}$ & $\mathrm{R}^{\mathrm{l}}$ & $\mathrm{R}^{2}$ & $\mathrm{R}^{3}$ & $\mathrm{R}^{4}$ & Solvent & $\mathbf{3}$ (yield) $^{\mathrm{a}}$ \\
\hline 1 & $\mathrm{Cr}$ & $p-\mathrm{CH}_{3} \mathrm{OC}_{6} \mathrm{H}_{4}$ & $\mathrm{CH}_{3}$ & $\mathrm{CH}_{3}$ & $\mathrm{CH}_{3}$ & $\mathrm{THF}$ & $\mathbf{3 a}(63)$ \\
2 & $\mathrm{Cr}$ & $p-\mathrm{CH}_{3} \mathrm{OC}_{6} \mathrm{H}_{4}$ & $\mathrm{CH}_{3}$ & $\mathrm{CH}_{3}$ & $\mathrm{CH}_{3}$ & Toluene & $\mathbf{3 a}(62)$ \\
3 & $\mathrm{Cr}$ & $p-\mathrm{CH}_{3} \mathrm{OC}_{6} \mathrm{H}_{4}$ & $\mathrm{CH}_{3}$ & $\mathrm{CH}_{3}$ & $\mathrm{CH}_{3}$ & Hexane & $\mathbf{3 a}(61)$ \\
4 & $\mathrm{Cr}$ & $p-\mathrm{CH}_{3} \mathrm{OC}_{6} \mathrm{H}_{4}$ & $\mathrm{CH}_{3}$ & $\mathrm{CH}_{3}$ & $\mathrm{CH}_{3}$ & DCE & $\mathbf{3 a}(82)$ \\
5 & $\mathrm{Cr}$ & $\mathrm{C}_{6} \mathrm{H}_{5}$ & $\mathrm{CH}_{3}$ & $\mathrm{CH}_{3}$ & $\mathrm{CH}_{3}$ & DCE & $\mathbf{3 b}(80)$ \\
6 & $\mathrm{Cr}$ & $p-\mathrm{ClC}_{6} \mathrm{H}_{4}$ & $\mathrm{CH}_{3}$ & $\mathrm{CH}_{3}$ & $\mathrm{CH}_{3}$ & DCE & $\mathbf{3 c}(91)$ \\
7 & $\mathrm{Cr}$ & $F_{2 r r o c e n y l}$ & $\mathrm{CH}_{3}$ & $\mathrm{CH}_{3}$ & $\mathrm{CH}_{3}$ & DCE & $\mathbf{3 d}(84)$ \\
8 & $\mathrm{Cr}$ & $\mathrm{C}_{6} \mathrm{H}_{5}$ & $\mathrm{Ment}^{\mathrm{c}}$ & $\mathrm{CH}_{3}$ & $\mathrm{CH}_{3}$ & DCE & $\mathbf{3 b}(95)$ \\
9 & $\mathrm{Cr}$ & $p-\mathrm{CH}_{3} \mathrm{OC}_{6} \mathrm{H}_{4}$ & $\mathrm{CH}_{3}$ & $\mathrm{C}_{6} \mathrm{H}_{5}$ & $\mathrm{C}_{6} \mathrm{H}_{5}$ & DCE & $\mathbf{3 a}(58)$ \\
10 & $\mathrm{Cr}$ & $\mathrm{C}_{6} \mathrm{H}_{5}$ & $\mathrm{CH}_{3}$ & $\mathrm{C}_{6} \mathrm{H}_{5}$ & $\mathrm{C}_{6} \mathrm{H}_{5}$ & DCE & $\mathbf{3 b}(65)$ \\
11 & $\mathrm{Cr}$ & $\mathrm{C}_{6} \mathrm{H}_{5}$ & $\mathrm{CH}_{3}$ & $\mathrm{C}_{6} \mathrm{H}_{5}$ & $\mathrm{H}$ & DCE & $-{ }^{\mathrm{d}}$ \\
12 & $\mathrm{~W}$ & $\mathrm{C}_{6} \mathrm{H}_{5}$ & $\mathrm{CH}_{3}$ & $\mathrm{CH}_{3}$ & $\mathrm{CH}_{3}$ & DCE & $\mathbf{3 b}(54)$ \\
\hline
\end{tabular}

${ }^{a}$ Isolated yields based on alkenyl carbene complexes $\mathbf{1}$.

${ }^{\mathrm{b}} \mathrm{DCE}=1,2$-dichloroethane.

${ }^{\mathrm{c}}$ Ment $=(1 R, 2 S, 5 R / 1 S, 2 R, 5 S)$-Menthyl.

${ }^{\mathrm{d}}$ A complex mixture of compounds was obtained. 
At this point it was desired to set up the conditions to stop the reaction at the alkoxydiene stage. This was brought about by simply running the reaction under the above conditions (3 equiv of allene, DCE, $60^{\circ} \mathrm{C}, 2-5 \mathrm{~h}$ ) followed by solvent removal and chromatographic purification using $\mathrm{Al}_{2} \mathrm{O}_{3}$ and a 10:1 mixture of hexane-ethyl acetate as the eluent. The achiral and chiral dienes thus prepared are depicted in Table 2. First, 2-methoxy-1,3-butadienes 4a-c were synthesized in 79-84\% yield from the corresponding methoxycarbene complexes 1 and 1,1dimethylallene 2a (entries 1-3). Interestingly, this methodology allowed for the efficient preparation of racemic and nonracemic chiral dienes 4d-f from carbene complexes derived from chiral alcohols, like ( \pm )-menthol (entries 4 and 5) and (-)-8-phenylmenthol (entry 6). In all the cases the $E$-diene derivatives $\left(\mathrm{J}_{\mathrm{H}(3)-\mathrm{H}(4)}=15-16 \mathrm{~Hz}\right.$ ) were obtained with excellent yields (90$94 \%$ ) and without $E / Z$ isomerization.

Table 2. Synthesis of 2-alkoxy-1,3-butadienes $\mathbf{4}$ from chromium alkenylcarbene complexes $\mathbf{1}$ and dimethylallene $\mathbf{2 a}$<smiles>[R]C=CC(=C)O[R2]</smiles>

1

$2 a$

4

\begin{tabular}{llll}
\hline Entry & $\mathrm{R}^{1}$ & $\mathrm{R}^{2}$ & $\mathbf{4}($ yield) \\
\hline 1 & $p-\mathrm{CH}_{3} \mathrm{OC}_{6} \mathrm{H}_{4}$ & $\mathrm{CH}_{3}$ & $\mathbf{4 a}(79)$ \\
2 & $\mathrm{C}_{6} \mathrm{H}_{5}$ & $\mathrm{CH}_{3}$ & $\mathbf{4 b}(84)$ \\
3 & $p-\mathrm{ClC}_{6} \mathrm{H}_{4}$ & $\mathrm{CH}_{3}$ & $\mathbf{4 c}(81)$ \\
4 & $\mathrm{C}_{6} \mathrm{H}_{5}$ & $\mathrm{Ment}^{\mathrm{b}}$ & $\mathbf{4 d}(90)$ \\
5 & $2-\mathrm{Furyl}_{6}$ & Ment $^{\mathrm{b}}$ & $\mathbf{4 e}(92)$ \\
6 & $\mathrm{C}_{6} \mathrm{H}_{5}$ & PhMent $^{\mathrm{b}}$ & $\mathbf{4 f}(94)$ \\
\hline
\end{tabular}

${ }^{a}$ Isolated yields from alkenyl carbene complexes $\mathbf{1}$.

${ }^{\mathrm{b}}$ Ment $=(1 R, 2 S, 5 R / 1 S, 2 R, 5 S)-$ Menthyl, PhMent $=(1 R, 2 S, 5 R)-8$-Phenylmenthyl.

The formation of compounds 4 can be explained by the chemo- and regioselective [2+2]cycloaddition between the $\mathrm{M}=\mathrm{C}$ function and the less substituted allene $\mathrm{C}=\mathrm{C}$ double-bond to form the metallacyclobutane intermediate I (Scheme 3). The latter would evolve through a retro $[2+2]$-cycloaddition to provide the 2-alkoxy-1,3-butadiene structure 4 . The accompanying metal vinylidene species II could be neither isolated nor trapped with alcohols. It is noteworthy that this metathesis reaction is a rather unusual process for alkoxy carbenes. ${ }^{12}$ Moreover, it represents 
a new reaction pathway for Fischer carbene complexes towards allenes which differs from those previously reported. ${ }^{10}$
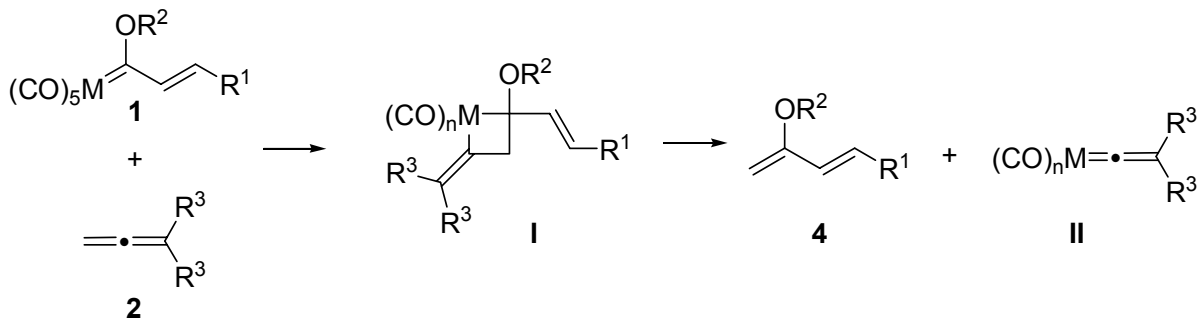

Scheme 3. Mechanism for the formation of alkoxydienes 4 from alkenyl carbene complexes $\mathbf{1}$ and allenes 2 .

In conclusion, we have developed a new methylenation reaction of alkenyl Fischer carbene complexes based on a rather unusual metathesis reaction with allenes. From a synthetic point of view, this approach provides an appropriate entry into useful achiral and chiral building blocks, such (E)-4-substituted-2-alkoxy-1,3-carbodienes. The availability and handling of the starting materials and the ease of the experimental make this one-step procedure preferable over those previously reported.

\section{Experimental Section}

General Procedures. ${ }^{1} \mathrm{H}$ NMR and ${ }^{13} \mathrm{C}$ NMR spectra were recorded at room temperature in $\mathrm{CDCl}_{3}$, on a Bruker AC-300 (300 and $75.5 \mathrm{MHz}$ ) and Bruker AMX-400 (400 and $\left.100 \mathrm{MHz}\right)$ spectrometers. Chemical shifts are given in ppm relative to TMS $\left({ }^{1} \mathrm{H}, 0.0 \mathrm{ppm}\right)$ or $\mathrm{CDCl}_{3}\left({ }^{13} \mathrm{C}\right.$, $77.0 \mathrm{ppm}$ ). Carbon multiplicities were assigned by DEPT techniques. Elemental analyses were carried out on a Perkin-Elmer 2400 and Carlo Erba 1108 microanalyzers.

TLC was performed on aluminum-backed plates with silica gel 60 with $\mathrm{F}_{254}$ indicator. All reactions were carried out under nitrogen using standard Schlenck techniques. 1,2dichloroethane was distilled from $\mathrm{CaH}_{2}$. Fischer carbene complexes 1 and 1,1-diphenylallene were prepared according to literature methods. All other reagents and solvents used in this work were of the best commercial grade available and used without further purification.

General procedure for the thermal reaction of alkenyl Fischer carbene complexes 1 and allenes 2. Synthesis of enone derivatives 3 and alkoxydienes 4

A solution of the carbene complex $1(0.5 \mathrm{mmol})$ and 1,1-dimethylallene $(1.5 \mathrm{mmol})$ in DCE (5 $\mathrm{mL}$ ) was stirred in a sealed tube at $60^{\circ} \mathrm{C}$ until disapearance of the starting carbene complex (checked by TLC; $2-5 \mathrm{~h}$ ). The solvent was then removed under reduced pressure and final 
chromatographic purification of the resulting residue afforded compounds $3\left(\mathrm{SiO}_{2} ; 10: 1\right.$ mixture of hexane:ethyl acetate) or dienes $4\left(\mathrm{Al}_{2} \mathrm{O}_{3} ; 10: 1\right.$ mixture of hexane:ethyl acetate).

(E)-4-(4-Methoxyphenyl)-3-buten-2-one (3a). Yellowish oil, ${ }^{1} \mathrm{H}-\mathrm{NMR}\left(\mathrm{CDCl}_{3}\right)$ : 2.38 (s, 3H), $3.87(\mathrm{~s}, 3 \mathrm{H}), 6.63(\mathrm{~d}, J=16.2 \mathrm{~Hz}, 1 \mathrm{H}), 6.94(\mathrm{~d}, J=7.5 \mathrm{~Hz}, 2 \mathrm{H}), 7.47-7.53(\mathrm{~m}, 3 \mathrm{H}) ;{ }^{13} \mathrm{C}-\mathrm{NMR}$ $\left(\mathrm{CDCl}_{3}\right): 27.3\left(\mathrm{CH}_{3}\right), 55.3\left(\mathrm{CH}_{3}\right), 114.3(\mathrm{CH}), 124.9(\mathrm{CH}), 126.9(\mathrm{C}), 129.8(\mathrm{CH}), 143.1(\mathrm{CH})$, 161.5 (C), 198.3 (C). Anal. Calcd for $\mathrm{C}_{11} \mathrm{H}_{12} \mathrm{O}_{2}$ : C, 74.98; H, 6.86; Found: C, 75.04; H, 6.88.

(E)-4-Phenyl-3-buten-2-one (3b). Colorless oil, ${ }^{1} \mathrm{H}-\mathrm{NMR}\left(\mathrm{CDCl}_{3}\right): 2.41$ (s, 3H), 6.74 (d, $J=$ $16.2 \mathrm{~Hz}, 1 \mathrm{H}), 7.41-7.43(\mathrm{~m}, 3 \mathrm{H}), 7.51-7.56(\mathrm{~m}, 3 \mathrm{H}) ;{ }^{13} \mathrm{C}-\mathrm{NMR}\left(\mathrm{CDCl}_{3}\right): 27.5\left(\mathrm{CH}_{3}\right), 127.0(\mathrm{CH})$, $128.2(\mathrm{CH}), 128.9(\mathrm{CH}), 130.5(\mathrm{CH}), 134.3(\mathrm{C}), 143.4(\mathrm{CH}), 198.4(\mathrm{C})$. Anal. Calcd for $\mathrm{C}_{10} \mathrm{H}_{10} \mathrm{O}$ : C, 82.16; H, 6.89; Found: C, 82.07; H, 6.85.

(E)-4-(4-Chlorophenyl)-3-buten-2-one (3c). Yellowish oil, ${ }^{1} \mathrm{H}-\mathrm{NMR}\left(\mathrm{CDCl}_{3}\right): 2.41$ (s, 3H), $6.71(\mathrm{~d}, J=16.5 \mathrm{~Hz}, 1 \mathrm{H}), 7.39(\mathrm{~d}, J=8.2 \mathrm{~Hz}, 2 \mathrm{H}), 7.44-7.52(\mathrm{~m}, 3 \mathrm{H}) ;{ }^{13} \mathrm{C}-\mathrm{NMR}\left(\mathrm{CDCl}_{3}\right): 27.7$ $\left(\mathrm{CH}_{3}\right), 127.5(\mathrm{CH}), 129.3(\mathrm{CH}), 129.4(\mathrm{CH}), 132.9(\mathrm{C}), 136.4(\mathrm{C}), 141.8(\mathrm{CH}), 198.1(\mathrm{C})$. Anal. Calcd for $\mathrm{C}_{10} \mathrm{H}_{9} \mathrm{ClO}$ : C, 66.49; H, 5.02; Found: C, 66.61; H, 4.98.

(E)-4-Ferrocenyl-3-buten-2-one (3d). Orange oil, ${ }^{1} \mathrm{H}-\mathrm{NMR}\left(\mathrm{CDCl}_{3}\right): 2.29$ (s, 3H), 4.15 (s, 5H), $4.44(\mathrm{~m}, 2 \mathrm{H}), 4.50(\mathrm{~m}, 2 \mathrm{H}), 6.33(\mathrm{~d}, J=15.9 \mathrm{~Hz}, 1 \mathrm{H}), 7.42(\mathrm{~d}, J=15.9 \mathrm{~Hz}, 1 \mathrm{H}) ;{ }^{13} \mathrm{C}-\mathrm{NMR}$ $\left(\mathrm{CDCl}_{3}\right): 27.1\left(\mathrm{CH}_{3}\right), 68.7(\mathrm{CH}), 69.7(\mathrm{CH}), 71.2(\mathrm{CH}), 78.5(\mathrm{C}), 124.6(\mathrm{CH}), 145.1(\mathrm{CH}), 197.8$ (C). Anal. Calcd for $\mathrm{C}_{14} \mathrm{H}_{14} \mathrm{FeO}$ : C, 66.17; H, 5.55; Found: C, 66.32; H, 5.61.

(E)-3-Methoxy-1-(4-methoxyphenyl)buta-1,3-diene (4a). Yellowish oil, ${ }^{1} \mathrm{H}-\mathrm{NMR}\left(\mathrm{CDCl}_{3}\right)$ : $3.70(\mathrm{~s}, 3 \mathrm{H}), 3.81(\mathrm{~s}, 3 \mathrm{H}), 4.22(\mathrm{~s}, 2 \mathrm{H}), 6.45(\mathrm{~d}, J=16.1 \mathrm{~Hz}, 1 \mathrm{H}), 6.84-6.91(\mathrm{~m}, 3 \mathrm{H}), 7.37(\mathrm{~d}, J=$ $8.7 \mathrm{~Hz}, 2 \mathrm{H}) ;{ }^{13} \mathrm{C}-\mathrm{NMR}\left(\mathrm{CDCl}_{3}\right): 54.9\left(\mathrm{CH}_{3}\right), 55.3\left(\mathrm{CH}_{3}\right), 86.2\left(\mathrm{CH}_{2}\right), 114.1(\mathrm{CH}), 122.6(\mathrm{CH})$, $127.9(\mathrm{CH}), 128.2(\mathrm{CH}), 129.6(\mathrm{C}), 159.4(\mathrm{C}), 159.5(\mathrm{C})$. Anal. Calcd for $\mathrm{C}_{12} \mathrm{H}_{14} \mathrm{O}_{2}$ : C, 75.76; H, 7.42; Found: C, 75.69; H, 7.53.

(E)-3-Methoxy-1-phenylbuta-1,3-diene (4b). Colorless oil, ${ }^{1} \mathrm{H}-\mathrm{NMR}\left(\mathrm{CDCl}_{3}\right): 3.73$ (s, 3H), 4.29 (s, 2H), 6.59 (d, $J=16.0 \mathrm{~Hz}, 1 \mathrm{H}), 6.97$ (d, $J=16.0 \mathrm{~Hz}, 1 \mathrm{H}), 7.25-7.47(\mathrm{~m}, 5 \mathrm{H}) ;{ }^{13} \mathrm{C}-\mathrm{NMR}$ $\left(\mathrm{CDCl}_{3}\right): 54.5\left(\mathrm{CH}_{3}\right), 86.9\left(\mathrm{CH}_{2}\right), 124.5(\mathrm{CH}), 126.5(\mathrm{CH}), 127.5(\mathrm{CH}), 128.4(\mathrm{CH}), 128.6(\mathrm{CH})$, 136.6 (C), 159.1 (C). Anal. Calcd for $\mathrm{C}_{11} \mathrm{H}_{12} \mathrm{O}: \mathrm{C}, 82.46$; H, 7.55; Found: C, 82.41; H, 7.56.

(E)-1-(4-Chlorophenyl)-3-methoxybuta-1,3-diene (4c). Yellowish oil, ${ }^{1} \mathrm{H}-\mathrm{NMR}\left(\mathrm{CDCl}_{3}\right): 3.70$ $(\mathrm{s}, 3 \mathrm{H}), 4.28(\mathrm{~s}, 2 \mathrm{H}), 6.52(\mathrm{~d}, J=15.8 \mathrm{~Hz}, 1 \mathrm{H}), 6.88(\mathrm{~d}, J=15.8 \mathrm{~Hz}, 1 \mathrm{H}), 7.28(\mathrm{~d}, J=8.5 \mathrm{~Hz}$, 2H), $7.34(\mathrm{~d}, J=8.5 \mathrm{~Hz}, 2 \mathrm{H}) ;{ }^{13} \mathrm{C}-\mathrm{NMR}\left(\mathrm{CDCl}_{3}\right): 54.9\left(\mathrm{CH}_{3}\right), 87.7\left(\mathrm{CH}_{2}\right), 125.2(\mathrm{CH}), 127.4$ $(\mathrm{CH}), 127.8(\mathrm{CH}), 128.7(\mathrm{CH}), 133.3(\mathrm{C}), 135.3(\mathrm{C}), 158.9(\mathrm{C})$. Anal. Calcd for $\mathrm{C}_{11} \mathrm{H}_{11} \mathrm{ClO}$ : C, 67.87; H, 5.70; Found: C, 67.93; H, 5.58.

(E) $(1 R, 2 S, 5 R / 1 S, 2 R, 5 S)-3-M e n t h y l o x y-1-p h e n y l b u t a-1,3-d i e n e ~(4 d)$. Yellowish oil, ${ }^{1} \mathrm{H}-$ NMR $\left(\mathrm{CDCl}_{3}\right): 0.92(\mathrm{~d}, J=6.9 \mathrm{~Hz}, 3 \mathrm{H}), 1.05-1.27(\mathrm{~m}, 8 \mathrm{H}), 1.53-1.84(\mathrm{~m}, 5 \mathrm{H}), 2.33(\mathrm{~m}, 2 \mathrm{H})$, $4.01(\mathrm{~m}, 1 \mathrm{H}), 4.33(\mathrm{~s}, 2 \mathrm{H}), 6.66(\mathrm{~d}, J=16.0 \mathrm{~Hz}, 1 \mathrm{H}), 6.91(\mathrm{~d}, J=16.0 \mathrm{~Hz}, 1 \mathrm{H}), 7.23-7.56(\mathrm{~m}$, $5 \mathrm{H}) ;{ }^{13} \mathrm{C}-\mathrm{NMR}\left(\mathrm{CDCl}_{3}\right): 16.8\left(\mathrm{CH}_{3}\right), 20.7\left(\mathrm{CH}_{3}\right), 22.2\left(\mathrm{CH}_{3}\right), 23.8\left(\mathrm{CH}_{2}\right), 26.3(\mathrm{CH}), 31.4(\mathrm{CH})$, 34.6 $\left(\mathrm{CH}_{2}\right), 39.6\left(\mathrm{CH}_{2}\right), 47.9(\mathrm{CH}), 76.4(\mathrm{CH}), 87.6\left(\mathrm{CH}_{2}\right), 125.9(\mathrm{CH}), 126.6(\mathrm{CH}), 127.5(\mathrm{CH})$, $128.3(\mathrm{CH}), 128.5(\mathrm{CH}), 136.9(\mathrm{C}), 157.0(\mathrm{C})$. Anal. Calcd for $\mathrm{C}_{20} \mathrm{H}_{28} \mathrm{O}: \mathrm{C}, 84.45$; H, 9.92; Found: C, 84.56; H, 9.85. 


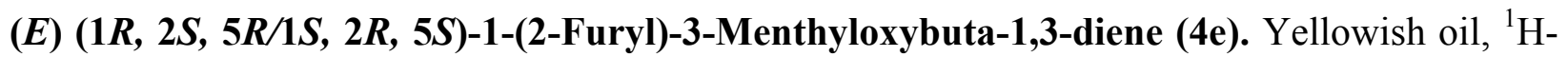
NMR $\left(\mathrm{CDCl}_{3}\right)$ : 0.92-1.76 (m, 16H), $2.45(\mathrm{~m}, 2 \mathrm{H}), 4.06(\mathrm{~m}, 1 \mathrm{H}), 4.39(\mathrm{~s}, 1 \mathrm{H}), 4.40(\mathrm{~s}, 1 \mathrm{H}), 6.23$ $(\mathrm{m}, 2 \mathrm{H}), 6.91(\mathrm{~d}, J=15.7 \mathrm{~Hz}, 1 \mathrm{H}), 7.25(\mathrm{~d}, J=15.7 \mathrm{~Hz}, 1 \mathrm{H}), 7.38(\mathrm{~s}, 1 \mathrm{H}) ;{ }^{13} \mathrm{C}-\mathrm{NMR}\left(\mathrm{CDCl}_{3}\right)$ : $17.0\left(\mathrm{CH}_{3}\right), 20.8\left(\mathrm{CH}_{3}\right), 22.3\left(\mathrm{CH}_{3}\right), 24.1\left(\mathrm{CH}_{2}\right), 26.7(\mathrm{CH}), 31.4(\mathrm{CH}), 34.8\left(\mathrm{CH}_{2}\right), 39.8\left(\mathrm{CH}_{2}\right)$, $48.3(\mathrm{CH}), 76.4(\mathrm{CH}), 88.1\left(\mathrm{CH}_{2}\right), 109.3(\mathrm{CH}), 111.8(\mathrm{CH}), 116.9(\mathrm{CH}), 125.0(\mathrm{CH}), 142.4$ (CH), 153.4 (C), 157.1 (C). Anal. Calcd for $\mathrm{C}_{18} \mathrm{H}_{26} \mathrm{O}_{2}$ : C, 78.79; H, 9.55; Found: C, 78.91; H, 9.53 .

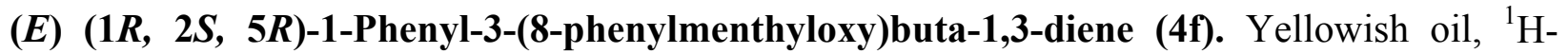
NMR $\left(\mathrm{CDCl}_{3}\right): 0.7(\mathrm{~d}, 3 \mathrm{H}), 0.93-1.21(\mathrm{~m}, 6 \mathrm{H}), 1.36(\mathrm{~s}, 3 \mathrm{H}), 1.50(\mathrm{~s}, 3 \mathrm{H}), 2.07(\mathrm{~m}, 1 \mathrm{H}), 2.33(\mathrm{~m}$, $1 \mathrm{H}), 4.10(\mathrm{~m}, 1 \mathrm{H}), 4.21(\mathrm{~s}, 1 \mathrm{H}), 4.33(\mathrm{~s}, 1 \mathrm{H}), 6.52(\mathrm{~d}, J=15.9 \mathrm{~Hz}, 1 \mathrm{H}), 6.74(\mathrm{~d}, J=15.9 \mathrm{~Hz}$, $1 \mathrm{H}), 7.06-7.45(\mathrm{~m}, 10 \mathrm{H}) ;{ }^{13} \mathrm{C}-\mathrm{NMR}\left(\mathrm{CDCl}_{3}\right): 22.7\left(\mathrm{CH}_{3}\right), 27.4\left(\mathrm{CH}_{3}\right), 28.0\left(\mathrm{CH}_{3}\right), 28.6\left(\mathrm{CH}_{3}\right)$, $32.0(\mathrm{CH}), 35.8\left(\mathrm{CH}_{2}\right), 40.4\left(\mathrm{CH}_{2}\right), 40.9(\mathrm{C}), 52.4(\mathrm{CH}), 77.4(\mathrm{CH}), 88.0\left(\mathrm{CH}_{2}\right), 126.2(\mathrm{CH})$, $126.8(\mathrm{CH}), 127.9(\mathrm{CH}), 128.4(\mathrm{CH}), 128.5(\mathrm{CH}), 128.9(\mathrm{CH}), 129.5(\mathrm{CH}), 129.8(\mathrm{CH}), 138.1$ (C), 152.1 (C), 157.0 (C). Anal. Calcd for $\mathrm{C}_{26} \mathrm{H}_{32} \mathrm{O}$ : C, 86.62; H, 8.95; Found: C, 86.73; H, 8.91.

\section{Acknowledgements}

We are grateful to the Ministerio de Educación y Ciencia (Project CTQ2004-08077), the Principado de Asturias (Project IB 05-136) and the Fundación Ramón Areces for the support of this research. We acknowledge the MEC for a predoctoral fellowship to R.V.

\section{References}

1. Reviews: (a) Petrzilka, M.; Grayson, J. I. Synthesis 1983, 753. (b) Schmidt, R. R. Acc. Chem. Res, 1986, 19, 250. (c) Barluenga, J.; Suárez-Sobrino, A.; López, L. A. Aldrichimica Acta 1999, 32, 4.

2. (a) Barluenga, J.; Aznar, F.; Valdés, C.; Cabal, M.-P. J. Org. Chem. 1991, 56, 6166. (b) Enders, D.; Meyer, O.; Raabe, G. Synthesis 1992, 1242. (c) Enders, D.; Meyer, O.; Raabe, G.; Runsink, J. Synthesis 1994, 66. (d) Enders, D.; Hecker, P.; Meyer, O. Tetrahedron 1996, $52,2909$.

3. Classical preparations of 2-alkoxy-1,3-dienes involve $O$-alkylation of dienolates and pyrolytic dealcoholysis of $\beta$-alkoxy acetals derived from $\alpha, \beta$-unsaturated carbonyl compounds: Dolby, L. J.; Marshall, K. Org. Prep. Proced. 1969, 1, 229. More recently, the methylenation of $\alpha, \beta$-unsaturated esters has been described: Petasis, N. A.; Lu, S.-P. Tetrahedron Lett. 1995, 2393, and references cited therein. For methodologies based on the palladium-catalyzed coupling of vinyl halides, see: Andersson, C.-M.; Hallberg, A. J. Org. Chem. 1989, 54, 1502, and references cited therein. 
4. (a) Barluenga, J.; Tomás, M.; Suárez-Sobrino, A.; López, L. A. Chem. Commun. 1995, 1785. (b) Barluenga, J.; Tomás, M.; López, L. A.; Suárez-Sobrino, A. Synthesis 1997, 967.

5. For recent reviews on Fischer carbene complexes, see: (a) Wulff, W. D. Comprehensive Organometallic Chemistry II, Vol 12 (Eds.: E. W. Abel, F. G. A. Stone, G. Wilkinson), Pergamon, New York, 1995; (b) Harvey, D. F.; Sigano, D. M. Chem. Rev., 1996, 96, 271; (c) Herndon, J. W. Tetrahedron, 2000, 56, 1257; (d) Barluenga, J; Fañanás, F. J. Tetrahedron, 2000, 56, 4597; (e) Sierra, M. A. Chem. Rev., 2000, 100, 3591; (f) Aumann, R. Eur. J. Org. Chem., 2000, 17; (g) de Meijere, A.; Schirmer, H.; Duestch, M. Angew. Chem., Int. Ed., 2000, 39, 3964; (h) Barluenga, J; Fernández-Rodríguez, M. A.; Aguilar, E. J. Organomet. Chem., 2005, 690, 539.

6. (a) Casey, C. P.; Betz, S. H. Tetrahedron Lett. 1973, 14, 1421. (b) Wulff, W. D.; Yang, D. C. J. Am. Chem. Soc. 1983, 105, 6726.

7. Barluenga, J.; Bernard, P. L. Jr; Concellón, J. M. Tetrahedron Lett. 1994, 35, 9471.

8. Barluenga, J.; Fernández-Marí, Viado, A. L.; Aguilar, E.; Olano, B.; García-Granda, S.; Moya-Rubiera, C. Chem. Eur. J. 1999, 5, 883. See also: Baldoli, C.; Del Buttero, P.; Licando, E.; Maiorana, S.; Papagni, A.; Zanotti-Gerosa, A. J. Organomet. Chem. 1994, 476, C27.

9. (a) Barluenga, J.; Bernad, P. L., Jr.; Concellón, J. M. Tetrahedron Lett. 1995, 36, 3937. (b) Barluenga, J.; Bernad, P. L., Jr.; Concellón, J. M.; Piñera-Nicolás, A.; García-Granda, S. J Org. Chem. 1997, 62, 6870.

10. For previous studies concerning the reactivity of simple Fischer carbene complexes with allenes, see: (a) Aumann, R.; Uphoff, J. Angew. Chem., Int. Ed. 1987, 26, 357. (b) Aumann, R.; Melchers, H. -D. J. Organomet. Chem. 1988, 355, 351. (c) Aumann, R.; Trentmann, B. Chem. Ber. 1989, 122, 1977. (d) Hwu, C.-C; Wang, F. C.; Yeh, M.-C. P.; Sheu, J.-H. J. Organomet. Chem. 1994, 474, 123

11. (a) Barluenga, J.; Vicente, R.; Barrio, P.; López, L. A.; Tomás, M. J. Am. Chem. Soc. 2004, 126, 5974. (b) Barluenga, J.; Vicente, R.; Barrio, P.; López, L. A.; Tomás, M.; Borge, J. J. Am. Chem. Soc. 2004, 126, 14354. (c) Barluenga, J.; Vicente, R.; López, L. A.; Tomás, M. J. Am. Chem. Soc. 2006, 128, 7050. (d) Barluenga, J; Vicente, R.; López, L. A.; Tomás, M. Tetrahedron 2005, 61, 11327.

12. (a) For a review on olefin metathesis initiated by Fischer carbene derivatives, see: Katz, T. J. Angew. Chem. Int. Ed. 2005, 44, 3010. (b) Hoffmann, M.; Buchert, M.; Reissig, H.-U. Chem. Eur. J. 1999, 5, 876. (c) For the synthesis of chromium aminocarbenes by metathesis with methoxy(phenyl)carbenes, see: Barluenga, J; Aznar, F.; Martín, A. Organometallics 1995, 14, 1429. (d) For the synthesis of chromium alkoxycarbenes by methatesis with diphenylcarbenechromium, see: Haase, W. C.; Nieger, M.; Dötz, K. H. Chem. Eur. J. 1999, 5, 2014. (e) For the intramolecular metathesis of vinylogous alkoxycarbenes, see: Watanuki, S.; Ochifuji, N.; Mori, M. Organometallics 1995, 14, 5062. 